\title{
The Inequalities of Positive Semi-Definite Block Matrix with
}

\section{Partial Order Relations}

\author{
Hui Quan* \\ Department of mathematics, Xiangtan University, Xiangtan411105, China
}

*Corresponding Author: Hui Quan, Department of mathematics, Xiangtan University, Xiangtan 411105, China

Abstract: In 2019, Zübeyde Ulukök obtained an important theorem in reference [1] : When $H=\left[\begin{array}{cc}A & C \\ C^{*} & B\end{array}\right]$ is a positive semi-definite matrix, then $H^{r} \leq 3\left[\lambda_{1}(A)+\lambda_{1}(B)\right]^{r-1}\left[\begin{array}{ll}A & \\ & B\end{array}\right]$, where $A, B$ are $n$-order square matrices. In this paper, we firstly do the same thing for a $3 \times 3$ positive semi-definite block matrix, and give a generalization of the above theorem. Next, we further generalize the case of $k \times k$ positive semi-definite block matrix, and discuss the partial ordering relationship between the sum of matrices on quasi-diagonal lines and block matrices at other locations. Thus, we gave a new eigenvalue inequality.

Keywords: Positive Semi-Definite Matrix Hermitian Matrix Partial Order Relation

\section{InTRODUCTION AND PRELIMINARIES}

Inequalities of positive semi-definite block matrices have been widely used in matrix theory. In recent years, inequalities about block matrices have become a hot topic of research. At the same time, some very good results have been obtained, such as references $[1,3,4,5]$. In this paper, we mainly discussed some positive semi-definite block matrices and obtained some matrix inequalities.

As we all know, the positive semi-definite block matrices have very good properties. Their eigenvalues are real numbers, so they can always be arranged in ascending order and we recorded then as $\lambda_{n}(A) \leq \cdots \leq \lambda_{2}(A) \leq \lambda_{1}(A)$. In this paper, we use symbol $\lambda_{1}(A)$ to represent the largest eigenvalue of a positive semi-definite matrix $A$. And use symbol $A \leq B$ to represent $B-A$ be a positive semi-definite matrix, obviously, " $\leq$ " is a partial order relation. In particular, $A \geq 0$ denotes that matrix $A$ is positive semi-definite. In addition, we call $U$ a unitary matrix if it satisfies $U^{*} U=I$, we call $A$ a Hermitian matrix if it satisfies $A^{*}=A$. Last, the $A \oplus B$ denotes the direct sum of $A$ and $B$, the block diagonal $\operatorname{matrix}\left[\begin{array}{cc}A & 0 \\ 0 & B\end{array}\right]$; and 0 represents a zero block matrix.

\section{Main Results}

Let's start with the following lemmas

Lemma1.1 Let $A \in M_{m, n}$ with $m \geq n$, then $\lambda\left(A A^{*}\right)=\lambda\left(A^{*} A \oplus 0\right)$ with $0 \in M_{m-n}$.

Lemma1.2 Let $A \in M_{n}$ be positive semi-definite matrix. Then $\lambda_{n}(A) I \leq A \leq \lambda_{1}(A) I$, where $I$ denotes 
the identity matrix in $M_{n}$.

Lemma1.3 If $\left(\begin{array}{ll}A & B \\ B & A\end{array}\right)$ is a positive semi-definite matrix, then $A \geq B$.

Proof Just consider the equation $A-B=\frac{1}{2}\left(\begin{array}{ll}I & -I\end{array}\right)\left(\begin{array}{ll}A & B \\ B & A\end{array}\right)\left(\begin{array}{c}I \\ -I\end{array}\right) \geq 0$ and we can get the conclusion.

Lemma1.4 If $\left(\begin{array}{cc}A & B \\ B^{*} & C\end{array}\right)$ is a positive semi-definite matrix, $A, C$ are square matrices of the same order, then we have $A+C \geq B+B^{*}$ and $A+C \geq-\left(B+B^{*}\right)$.

Proof Becase $\left(\begin{array}{cc}A & B \\ B^{*} & C\end{array}\right)$ is a positive semi-definite matrix, So, for any unitary matrix $U$,we have $U^{*}\left(\begin{array}{cc}A & B \\ B^{*} & C\end{array}\right) U \geq 0$, thus there is $\left(\begin{array}{cc}A & B \\ B^{*} & C\end{array}\right)+U^{*}\left(\begin{array}{cc}A & B \\ B^{*} & C\end{array}\right) U \geq 0$. In particular, take $U=\left(\begin{array}{ll}0 & I \\ I & 0\end{array}\right)$, then we have $\left(\begin{array}{cc}A+C & B+B^{*} \\ B+B^{*} & A+C\end{array}\right) \geq 0$. From lemma 1.3 we can get that $A+C \geq B+B^{*}$. And we can draw another conclusion by replacing the original $B$ with $-B$.

Lemma1.5 If $H \in M_{n}$ is a positive semi-definite matrix, then for any $n \times k$ unitary matrix $U$ satisfying $U^{*} U=I_{k}$ and for each $i=1, \ldots, k$, we have $\lambda_{i+m-k}(H) \leq \lambda_{i}\left(U^{*} H U\right) \leq \lambda_{i}(H)$.

Theorem $1 \mathbf{1}_{H}=\left[\begin{array}{ccc}A & D & E \\ D^{*} & B & F \\ E^{*} & F^{*} & C\end{array}\right]$ is a positive semi-definite matrix, where $A, B, C$ are all n-order square matrices, then

$H^{r} \leq 3\left[\lambda_{1}(A)+\lambda_{1}(B)+\lambda_{1}(C)\right]^{r-1}\left[\begin{array}{lll}A & & \\ & B & \\ & & C\end{array}\right]$ for $r \geq 1$.

ProofBecause $H$ is a positive semi-definite matrix, so there exists an invertible matrix $P$ such that $H=P^{*} P$. We divide $P$ into blocks: $P=[X, Y, Z]$, where $X, Y, Z \in M_{3 n \times n}$, and we can know that $X^{*} X=A, \quad Y^{*} Y=B, \quad Z^{*} Z=C$. From Lemma 1.1, we can get the following results: $\lambda_{1}\left(X X^{*}\right)=\lambda_{1}\left(X^{*} X \oplus 0\right)=\lambda_{1}(A), \lambda_{1}\left(Y Y^{*}\right)=\lambda_{1}\left(Y^{*} Y \oplus 0\right)=\lambda_{1}(B)$, $\lambda_{1}\left(Z Z^{*}\right)=\lambda_{1}\left(Z^{*} Z \oplus 0\right)=\lambda_{1}(C)$.

Noting that the following equation holds: $H^{r}=\left(P^{*} P\right)^{r}=P^{*}\left(P P^{*}\right)^{r-1} P$, and we denote 
$T=\left(P P^{*}\right)^{r-1}$. So, for $H^{r}$, there is the following decompositions :

$H^{r}=P^{*} T P=\left[\begin{array}{ccc}X^{*} T X & X^{*} T Y & X^{*} T Z \\ Y^{*} T X & Y^{*} T Y & Y^{*} T Z \\ Z^{*} T X & Z^{*} T Y & Z^{*} T Z\end{array}\right]=\left[\begin{array}{lll}X & & \\ & Y & \\ & & Z\end{array}\right]^{*}\left[\begin{array}{ccc}T & T & T \\ T & T & T \\ T & T & T\end{array}\right]\left[\begin{array}{lll}X & & \\ & Y & \\ & & Z\end{array}\right]$

We notice that $\left[\begin{array}{ccc}T & T & T \\ T & T & T \\ T & T & T\end{array}\right]=\left[\begin{array}{ccc}T^{\frac{1}{2}} & 0 & 0 \\ T^{\frac{1}{2}} & 0 & 0 \\ T^{\frac{1}{2}} & 0 & 0\end{array}\right]\left[\begin{array}{ccc}T^{\frac{1}{2}} & 0 & 0 \\ T^{\frac{1}{2}} & 0 & 0 \\ T^{\frac{1}{2}} & 0 & 0\end{array}\right]^{*}$, and $\lambda\left[\begin{array}{ccc}T & T & T \\ T & T & T \\ T & T & T\end{array}\right]=3 \lambda(T)$.

So, from lemma 1.2. We can get that:

$$
\begin{aligned}
& H^{r} \leq 3 \lambda_{1}(T)\left[\begin{array}{lll}
X & & \\
& Y & \\
& & Z
\end{array}\right]^{*}\left[\begin{array}{lll}
X & & \\
& Y & \\
& & Z
\end{array}\right]=3 \lambda_{1}(T)\left[\begin{array}{lll}
A & & \\
& B & \\
& & C
\end{array}\right]=3 \lambda_{1}\left(P P^{*}\right)^{r-1}\left[\begin{array}{lll}
A & & \\
& B & \\
& & C
\end{array}\right] \\
& =3 \lambda_{1}^{r-1}\left(X X^{*}+Y Y^{*}+Z Z^{*}\right)\left[\begin{array}{lll}
A & & \\
& B & \\
& & C
\end{array}\right] \leq 3\left[\lambda_{1}\left(X X^{*}\right)+\lambda_{1}\left(Y Y^{*}\right)+\lambda_{1}\left(Z Z^{*}\right)\right]^{r-1}\left[\begin{array}{lll}
A & & \\
& B & \\
& & C
\end{array}\right] \\
& =3\left[\lambda_{1}(A)+\lambda_{1}(B)+\lambda_{1}(C)\right]^{r-1}\left[\begin{array}{llll}
A & & \\
& B & \\
& & C
\end{array}\right] . \text { So, the theorem is proved. }
\end{aligned}
$$

On the basis of this conclusion, we can easily get the results of Zübeyde Ulukök in reference[1]:

Corollary $1 H=\left[\begin{array}{cc}A & C \\ C^{*} & B\end{array}\right]$ is a positive semi-definite matrix, where $A, B$ are n-order square matrices, then

$$
H^{r} \leq 3\left[\lambda_{1}(A)+\lambda_{1}(B)\right]^{r-1}\left[\begin{array}{ll}
A & \\
& B
\end{array}\right] \text { for } r \geq 1 .
$$

Next, we will discuss the case of $k \times k$ positive semi-definite block matrices and explore the relationship between quasi-diagonal matrices and other block matrices at other locations.

Theorem 2 $H=\left[\begin{array}{cccc}M_{11} & M_{12} & \cdots & M_{1 k} \\ M_{12}^{*} & M_{22} & \cdots & M_{2 k} \\ \vdots & \vdots & \ddots & \vdots \\ M_{1 k}^{*} & M_{2 k}^{*} & \cdots & M_{k k}\end{array}\right]$ is a positive semi-definite matrix, and $M_{11}, \ldots, M_{k k}$ are all n-order square matrices, if it satisfied $M_{i j}$ are all Hermitian matrices, for

$(1 \leq i \leq j \leq n)$, then

$$
\sum_{i=1}^{k} M_{i i} \geq \frac{2}{k-1} \sum_{1 \leq i<j \leq n} M_{i j}
$$

Proof First of all, notice the following facts: if $U_{1}, \ldots, U_{k}$ are all unitary matrices, $A$ is a positive semi-definite matrix, then $U_{1}^{*} A U_{1}+\ldots U_{k}^{*} A U_{k}$ is still a positive semi-definite matrix. 
After calculation, we can get that $\sum_{i=1}^{k-1} \sum_{l=i+1}^{k}\left(\begin{array}{ll}M_{i i} & M_{i l} \\ M_{i l} & M_{l l}\end{array}\right) \geq 0$. From lemma 1.4 we can get that : $\sum_{i=1}^{k-1} \sum_{l=i+1}^{k}\left(M_{i i}+M_{l l}\right) \geq \sum_{i=1}^{k-1} \sum_{l=i+1}^{k}\left(M_{i l}+M_{i l}\right)=2 \sum_{1 \leq i<j \leq n} M_{i j}$

So, there is $\sum_{i=1}^{k} M_{i i} \geq \frac{2}{k-1} \sum_{1 \leq i<j \leq n} M_{i j}$.

Corollary 2If $\left(\begin{array}{cc}A & B \\ B^{*} & C\end{array}\right)$ is a positive semi-definite matrix, $A, C$ are square matrices of the same order, and $B$ is a Hermitian matrix, then $\operatorname{tr} B \leq \max \{\operatorname{tr} A, \operatorname{tr} B\}$.

Theorem3 $H=\left[\begin{array}{cccc}M_{11} & M_{12} & \cdots & M_{1 k} \\ M_{12}^{*} & M_{22} & \cdots & M_{2 k} \\ \vdots & \vdots & \ddots & \vdots \\ M_{1 k}^{*} & M_{2 k}^{*} & \cdots & M_{k k}\end{array}\right]$ is a positive semi-definite matrix, and $M_{11}, \ldots, M_{k k}$ are all n-order square matrices, if it satisfied $M_{i j}$ are all Hermitian matrices, for $(1 \leq i<j \leq n)$, then for these $1 \leq i \leq n$, we have $\frac{2}{k} \lambda_{i}\left(\sum_{1 \leq i<j \leq n} M_{i j}\right) \leq \lambda_{i}(H)$

Proof Take a unitary matrix $U=\frac{1}{\sqrt{k}}\left[\begin{array}{c}I \\ I \\ \vdots \\ I\end{array}\right]$, then $U$ satisfied $U^{*} U=I_{n}$, noticed that $M_{i j}$ are all Hermitian matrices, for $(1 \leq i<j \leq n)$,so we have

$$
\begin{aligned}
U^{*} H U & =\frac{1}{k} \sum_{i=1}^{k} M_{i i}+\frac{2}{k} \sum_{1 \leq i<j \leq n} M_{i j}, \geq \frac{1}{k} \frac{2}{k-1} \sum_{1 \leq i<j \leq n} M_{i j}+\frac{2}{k} \sum_{1 \leq i<j \leq n} M_{i j} \quad \text { (from Theorem 2) } \\
& =\frac{2}{k} \sum_{1 \leq i<j \leq n} M_{i j}\left(\frac{1}{k-1}+1\right) \geq \frac{2}{k} \sum_{1 \leq i<j \leq n} M_{i j} .
\end{aligned}
$$

Then for $1 \leq i \leq n$, from lemma 1.5 we have $\frac{2}{k} \lambda_{i}\left(\sum_{1 \leq i<j \leq n} M_{i j}\right) \leq \lambda_{i}\left(U^{*} H U\right) \leq \lambda_{i}(H)$, thus the conclusion $\frac{2}{k} \lambda_{i}\left(\sum_{1 \leq i<j \leq n} M_{i j}\right) \leq \lambda_{i}(H)$ is proved.

\section{REFERENCES}

[1] Zübeyde Ulukök, More inequalities for positive semidefinite $2 \times 2$ block matrices and their blocks [J]. Linear Algebra and its Applications 572 (2019) 51-67

[2] X. Zhan, Matrix Inequalities, Lecture Notes in Math., vol. 1790, Springer-Verlag, Berlin, 2002. 
[3] Y. Zhang, Eigenvalue majorization inequalities for positive semidefinite block matrices and their blocks, Linear Algebra Appl. 446 (2014) 216-223.

[4] F. Zhang, Matrix inequalities by means of block matrices, Mathematical Inequalities and Applications, Vol. 4, No. 4, (2001), 481-490.

[5] R. Türkmen and Z. Ulukök, Inequalities for singular values of positive semidefinite block matrices[J]. International Mathematical Forum, Vol. 6, 2011, no. 31, 1535 - 1545

Citation: Hui Quan (2019).The Inequalities of Positive Semi-Definite Block Matrix with Partial Order Relations. International Journal of Scientific and Innovative Mathematical Research (IJSIMR), 7(8), pp. 3-7. http://dx.doi.org/ 10.20431/2347 -3142.0708002

Copyright: (C) 2019 Authors, this is an open-access article distributed under the terms of the Creative Commons Attribution License, which permits unrestricted use, distribution, and reproduction in any medium, provided the original author and source are credited. 